\title{
Better nasal recovery and intact olfactory function after transseptal approach for endoscopic endonasal transphenoidal adenomectomy: a retrospective analysis
}

\author{
Yen-Hui Lee ${ }^{1}$, Huan-Chih Wang ${ }^{1}$, Yi-Tsen Lin ${ }^{1}$, Shih-Hung Yang ${ }^{1}$, Chih-Feng Lin ${ }^{1}$, Po-Hao \\ Huang ${ }^{1}$, Kuo-Chuan Wang ${ }^{1}$, Dar-Ming Lai ${ }^{1}$, Ham-Min Tseng ${ }^{1}$, and Te-Huei Yeh ${ }^{1}$ \\ ${ }^{1}$ National Taiwan University Hospital
}

January 24, 2022

\begin{abstract}
Objectives Endoscopic endonasal transsphenoidal adenomectomy (TSA) is the most frequently performed skull base surgery and researchers have recently focused on preserving nasal function. The transseptal approach is a promising procedure owing to its less injury to nasal mucosa. Therefore, we conducted this study to investigate whether the transseptal approach could reduce nasal morbidities with comparable neurosurgical outcomes. Study design Retrospective chart review. Setting Singleinstitution tertiary care centre. Participants 25 patients of transseptal approach from January 2019 to December 2020 and 25 patients of transnasal approach from January 2017 to December 2018. Main outcome measures Lund-Kennedy endoscopic scores, 22-item sinonasal outcome test (SNOT-22) scores, TIB smell identification tests (TIBSITß) scores, the rate of gross total resection, endocrine control, and postoperative complications were collected. Results The postoperative SNOT-22 (4.40 vs. $7.36, \mathrm{P}=.005)$ and Lund-Kennedy endoscopic scores $(2.72$ vs, 5.08 at 2 weeks, 0.88 vs. 3.52 at 1 month, 0.48 vs. 2.12 at 3 months, 0.04 vs. 1.76 at 6 months, all adjusted $\mathrm{P}<.01)$ were significantly lower in the transseptal group, while the TIBSIT scores were not significantly different (41.6 vs. 42.4 preoperatively, $\mathrm{P}=.68 ; 41.6$ vs. 40.2 postoperatively, $\mathrm{P}=.46$ ). The rates of gross total resection, recovery of hormonal abnormalities, and complications were not significantly different. In multivariate analysis, the transseptal approach remained an independent factor for lower SNOT-22 scores and Lund-Kennedy endoscopic scores. Conclusion The transseptal approach provides better recovery of nasal mucosa and intact olfactory function without compromising neurosurgical outcomes.
\end{abstract}

Better nasal recovery and intact olfactory function after transseptal approach for endoscopic endonasal transphenoidal adenomectomy: a retrospective analysis

Running title: Better nasal recovery after endoscopic endonasal transseptal TSA

Keywords: Skull base surgery, Rhinology, smell

\section{ABSTRACT}

\section{Objectives}

Endoscopic endonasal transsphenoidal adenomectomy (TSA) is the most frequently performed skull base surgery and researchers have recently focused on preserving nasal function. The transseptal approach is a promising procedure owing to its less injury to nasal mucosa. Therefore, we conducted this study to investigate whether the transseptal approach could reduce nasal morbidities with comparable neurosurgical outcomes.

Study design Retrospective chart review. 
Setting Single-institution tertiary care centre.

Participants 25 patients receiving transseptal approach from January 2019 to December 2020 and another 25 patients undergoing transnasal approach from January 2017 to December 2018.

\section{Main outcome measures}

Lund-Kennedy endoscopic scores, 22-item sinonasal outcome test (SNOT-22) scores, Top International Biotech smell identification tests (TIBSIT@) scores, the rate of gross total resection, endocrine control, and postoperative complications were collected.

\section{Results}

Comparing the transseptal and transnasal groups, the postoperative SNOT-22 (4.40 vs. 7.36, $P=.005)$ and Lund-Kennedy endoscopic scores (2.72 vs, 5.08 at 2 weeks, 0.88 vs. 3.52 at 1 month, 0.48 vs. 2.12 at 3 months, 0.04 vs. 1.76 at 6 months, all adjusted $P<.01$ ) were significantly lower in the transseptal group, while the TIBSIT scores were not significantly different (41.6 vs. 42.4 preoperatively, $P=.68 ; 41.7$ vs. 40.2 postoperatively, $P=$.46). The rates of gross total resection, recovery of hormonal abnormalities, and complications were not significantly different. In multivariate analysis, the transseptal approach remained an independent factor for lower SNOT-22 scores and Lund-Kennedy endoscopic scores.

\section{Conclusion}

The transseptal approach provides better recovery of nasal mucosa and intact olfactory function without compromising neurosurgical outcomes.

\section{Key points}

- The transseptal approach for endoscopic endonasal transsphenoidal adenomectomy (TSA) is a promising procedure owing to its less injury to nasal mucosa. However, because limited space may compromise resection, whether it is a worthy trade-off remains to be validated and no studies reported simultaneously both rhinological and neurosurgical outcomes.

- This study revealed that the postoperative SNOT-22 and Lund-Kennedy endoscopic scores were significantly lower in the transseptal group than in the transnasal group. The olfactory function test using TIBSIT revealed no significant difference between the two groups.

- The neurosurgical outcomes, including gross total resection, hormone control and hospital stay, were similar between the two groups. The rate of complications were not significantly different between the two groups.

- After controlling all possible confounding factors, transseptal approach remained an independent factors of lower SNOT-22 scores and Lund-Kennedy scores.

- The endoscopic transseptal TSA provides better recovery of nasal mucosa and intact olfactory function without compromising neurosurgical outcomes.

\section{INTRODUCTION}

Transsphenoidal adenomectomy (TSA) has evolved enormously and is the most frequently performed skull base surgery nowadays. ${ }^{1}$ The endoscopic endonasal approach (EEA) has become a popular surgical method for TSA because of its excellent visualization and minimal comorbidities. Since EEA is a mature and established surgical approach with minimal complications, researchers have recently turned their focus on preserving nasal function and quality of life.

Many otolaryngologists wonder whether rhinological outcome can be altered by choosing different surgical corridors for TSA, which are classified according to the routes of sphenoidotomy. ${ }^{2}$ The most common route of sphenoidotomy is transnasal, namely dissection directly from the bilateral sphenoidoethmoidal recess in the nasal cavity. Although it seems straightforward, it inevitably damages the anterior wall of the sphenoid sinus, the posterior nasal septum, and their overlying mucosa. In contrast, transseptal sphenoidotomy, defined as dissection through the subperichondrial and subperiosteal planes of the nasal septum, 
preserves the nasal mucosa and restores the integrity of the sphenoid sinus and posterior nasal septum. The transseptal approach, via either the endonasal or the sublabial route, was once the state-of-the-art surgical method performed in microscopic TSA. ${ }^{3}$ Recently, several groups have undertaken efforts to develop endoscopic endonasal transseptal TSA and have reported promising rhinological results. ${ }^{4-8}$ Endoscopic endonasal transseptal TSA should receive more attention during the coronavirus disease 2019 (COVID-19) pandemic because the integrity of the sphenoid sinus provents unexpected injuries from common nasal procedures, such as nasopharyngeal swab test or nasogastric tube placement, ${ }^{9,}{ }^{10}$ for the rest of their lives.

However, endoscopic endonasal transseptal TSA might be questioned due to the limitations of the maneuverability and exposure. Several studies have reported solely the neurosurgical outcomes, ${ }^{4,5}$ while no studies have compared rhinological and neurosurgical outcomes concurrently to delineate the tradeoff. Therefore, we conducted this study to investigate whether the transseptal approach for endoscopic endonasal TSA could prevent nasal morbidities without compromising neurosurgical outcomes.

\section{MATERIALS AND METHODS}

Patients

Our surgical teams started performing transseptal approach since January, 2019 for all patients with pituitary tumor, except for those with (1) history of previous sphenoid surgery, transnasal TSA or septoplasty. (2) Pathology other than pituitary adenoma suspected on imaging. We reviewed all patients receiving transseptal approach from January 2019 to December 2020 and excluded the patients with following criteria: (1) histopathology other than pituitary adenoma; (2) concurrent sinonasal disease; (3) positive result of polymerase chain reaction (PCR) test for severe acute respiratory syndrome coronavirus 2 (SARS-CoV-2) at any time of observation (4) harvest of a nasoseptal flap.

Of 35 consecutive adult patients ([?] 20 years old) receiving transseptal approach, 10 (29\%) patients were excluded for the following reasons: $7(20 \%)$ had different pathology other than pituitary adenoma, at $2(6 \%)$ had chronic sinusitis simultaneously, $1(3 \%)$ had positive PCR test result for SARS-CoV-2. A total of 25 patients were included for analysis (transseptal group). Another 25 consecutive patients receiving endoscopic endonasal transnasal TSA from January 2017 to December 2018 fulfilling the same inclusion/exclusion criteria were selected as control (transnasal group).

This study was approved by the institutional review board of our hospital (IRB: 202011002RINA) and followed the Strengthening the Reporting of Observational Studies in Epidemiology guideline.

\section{Preoperative assessment}

Before surgery, all patients underwent detailed history taking, radiological studies, endocrinological function tests. Radiological studies encompassed magnetic resonance imaging (MRI) with a pituitary protocol and fine-cut computerised tomography. The size of the tumor was defined as the maximal diameter measured on coronal view and regional invasion of the tumor was also recorded. We evaluated the endocrinological function with a complete hormone battery of the anterior pituitary lobe and the functional status could thus be classified as normal, hypersecreting and hypopituitarism.

In addition, we performed endoscopic examination preoperatively to rule out sinonasal disease. To evaluate baseline olfactory function, we used the Top International Biotech Smell Identification Test (TIBSIT(r), Top International Biotech, Neihu, Taipei, Taiwan), which substitutes odors more familiar to the Taiwanese population for certain odors in the traditional Chinese version of the University of Pennsylvania Smell Identification Test. ${ }^{12}$

\section{Surgical techniques: Endoscopic transseptal approach (Video 1)}

After lateralization of bilateral turbinates, the first incision was made anterior to the nasal septal body to avoid bleeding. The mucosal flap was elevated from the subperichondrial and continuing subperiosteal planes, followed by dissection superiorly to the perpendicular plate of the ethmoid bone, posteriorly to the sphenoid rostrum, laterally to the sphenoid sinus ostium, and inferiorly to the nasal floor. Another incision on the 
other side was made posterior to the nasal septal body to avoid perforation, followed by the same dissection. Partial removal of the perpendicular plate was performed, and we applied a Cottle nasal speculum between the septal flaps to establish a surgical corridor and to prevent unexpected injury to the septal mucosa and olfactory neuroepithelium (Fig. 1A and 1B). Then we performed sphenoidotomy by removing the rostrum. After creating a sufficient space for four-hand surgery, neurosurgeons removed the tumor until the arachnoid membrane descended (Fig. 1C and 1D). Finally, we repositioned the septal flaps and restore bilateral middle turbinates to the neutral position to prevent sinusitis. Bilateral incision wounds were left unsutured to facilitate drainage of exudate.

\section{Surgical techniques: Endoscopic transnasal approach}

In transnasal group, the mucosa, the bony structures of the posterior nasal septum and the anterior wall of the sphenoid sinuses were removed. Then the tumor was resected in the same manner. At the end of the surgery, sphenoid sinuses were left wide open and the posterior septectomy window remained.

\section{Neurosurgical outcomes}

We collected the length of hospital stay, rate of total resection and rate of alteration in pituitary hormone as neurosurgical outcomes. Gross total resection was defined as the absence of visible residual tumor on follow-up MRI 6 months after the operation. We defined the postoperative alteration of hormone status at 6 months after surgery as: (1) improved: any of the abnormal axes became normal or closer to the normal value; (2) stationary: the abnormal axes remained unchanged; or (3) worsened: the abnormal axes became more deviated from the normal value.

\section{Rhinological outcomes}

We quantified the recovery of nasal mucosa 2 weeks, 1 month, 3 months and 6 months after surgery using he Lund-Kenney endoscopic scoring system. At 6 months, we performed SNOT-22, which was already validated as an effective tool to monitor nasal symptoms after endoscopic skull base surgery. ${ }^{11}$ Also, we repeated TIBSIT(r) and classified the olfactory status according to the normative values for different age and gender groups. ${ }^{12}$

\section{Complications}

Diabetes insipidus, postoperative CSF leakage, hypopituitarism, meningitis, and intracranial hemorrhage were recorded as neurosurgical complications. Epistaxis, chronic sinusitis, and septal infection were recorded as nasal complications.

\section{Statistical analysis}

Student's t test and Chi-square test/Fisher's exact test were used for comparisons of characteristics of the two groups. The sequential changes in Lund-Kennedy scores were analysed with repeated measures ANOVA, followed by comparisons between groups at the 4 specified timepoints using Student's t test with Bonferroni correction. The effect of transseptal approach on SNOT-22 scores and Lund-Kennedy scores was further analysed using linear regression and generalised estimating equations. A $P$ value less than .05 was considered statistically significant. All of the analyses were conducted with R, version 4.0.2.

\section{RESULTS}

\section{Clinical characteristics}

The demographics, radiological features and endocrine functions are summarised in Table 1. The mean ages of the transseptal and transnasal group were $59.2+-14.6$ and $56.3+-15.8$ years old. There were 11 male and 14 female subjects in the transseptal group, and 14 male and 11 female subjects in the transnasal group. Age and sex were not significantly different between the two groups.

The mean tumor size in the transseptal group was $2.36+-0.79 \mathrm{~cm}$, while that in the transnasal group was $2.32+-0.86 \mathrm{~cm}$. The numbers of lesions classified by Hardy classification from I to IV were $0(0 \%)$, 
$12(48 \%), 9(36 \%)$ and $4(16 \%)$ in the transseptal group and $4(16 \%), 9(36 \%), 10(40 \%)$ and $2(8 \%)$ in the transnasal group. The number of tumors with regional invasiveness, that is, parasellar, suprasellar and infrasellar extension, were $23(92 \%), 21(84 \%)$, and $4(16 \%)$, respectively, in the transseptal group and 19 $(76 \%), 19(76 \%)$, and $10(40 \%)$, respectively, in the transnasal group. None of the radiological features were significantly different between the two groups.

Preoperative hypopituitarism was noted only in $4(16 \%)$ patients in both groups. Five (20\%) patients in the transseptal group and $9(36 \%)$ patients in the transnasal group had hypersecretion of hormones. The preoperative hormonal statuses and the distribution of hypersecreting hormones were not significantly different between the two groups.

Neurosurgical outcomes

The neurosurgical outcomes were summarised in Table 2. The mean hospital stay was $6.24+-1.92$ days in the transseptal group and $7.72+-3.58$ days in the transnasal group. Gross total resection was obtained in $14(56 \%)$ patients in the transseptal group and $15(60 \%)$ patients in the transnasal group. The hormonal status improved in $8(89 \%)$ patients with preoperative hormone abnormalities in the transseptal group and $12(92 \%)$ patients in the transnasal group. There was no significant difference in hospital stay, gross total resection rate and hormone control between these two groups.

\section{Nasal outcomes}

The postoperative Lund-Kennedy scores, SNOT-22 scores and TIBSIT scores were summarised in Fig. 2. Postoperative Lund-Kennedy score was significantly lower in the transseptal group than in the transnasal group $(P<$. 001). Post-hoc analysis revealed that the Lund-Kennedy scores were also lower at every timepoint after surgery ( 2.72 vs, 5.08 at 2 weeks, 0.88 vs. 3.52 at 1 month, 0.48 vs. 2.12 at 3 months, 0.04 vs. 1.76 at 6 months, all adjusted $P<.01$ ). SNOT-22 showed also a lower postoperative scores in the transseptal group than in the transsnasal group at 6 months after surgery (4.40 vs. $7.36, P=$. 005). Nevertheless, the TIBSIT scores were not significantly different between the two approaches (41.6 vs. 42.4 preoperatively, $P=.68 ; 41.6$ vs. 40.2 postoperatively, $P=$. 46). The olfactory status based on normal values of different gender and age remained unchanged for all patients.

Table 3 showed the effect of transseptal approach on the postoperative Lund-Kennedy scores and SNOT-22 scores using regression models. The univariate model revealed a significant lower Lund-Kennedy scores and SNOT-22 scores in the transseptal group ( $\beta,-2.09 ; 95 \%$ confidence interval $(\mathrm{CI}),(-2.54,-1.64), P<.001$ and $\beta,-2.96 ; 95 \%$ CI, (-4.98, -0.93), $P=.005$, respectively). After controlling age, sex, Hardy grade, presence of regional invasion and endocrine function, transseptal approach remains an independent factor for lower Lund-Kennedy scores and SNOT-22 scores $(\beta,-2.24 ; 95 \%$ CI, $(-2.73,-1.75), P<.001$ and $\beta,-2.46 ; 95 \%$ CI, $(-4.60,-0.93), P=.02$, respectively).

\section{Complications}

Postoperative complications are summarised in Table 4. The rates of neurosurgical complications, including transient diabetes insipidus, hypopituitarism, and postoperative CSF leakage were not significantly different between the two groups ( $P=.77,1.00$ and 1.00 , respectively). No patients had meningitis, prolonged diabetes insipidus or intracranial hemorrhage.

One patient (4\%) in each group reported self-limiting epistaxis. One (4\%) patient in the transseptal group was found to have septal infection and was treated successfully with short-term antibiotics. There was no significant difference in these two complications between the two groups ( $P=1.00$ and 1.00, respectively). None of the patients in the transseptal group had postoperative septal perforation.

\section{DISCUSSION}

Our study revealed that the transseptal approach had better patient-reported outcomes and objective endoscopic scores with intact olfactory function. Meanwhile, the neurosurgical outcomes and rate of complications 
were comparable. That is, endoscopic transseptal TSA facilitates nasal recovery without compromising neurosurgical outcomes.

In recent years, neurosurgeons and otolaryngologists have been making a joint effort to reduce nasal morbidities. ${ }^{13-17}$ There is increasing evidence indicating that endoscopic transnasal TSA has better neurosurgical outcomes and fewer complications than microscopic transsetpal TSA, ${ }^{18-20}$ while the sinonasal outcomes have been inconsistent. ${ }^{7,21,22}$ Interestingly, Hong et al. found better sinonasal quality of life in the early postoperative period in the microscopic transseptal group than in the endoscopic transnasal group. ${ }^{7}$ They proposed that their finding was because they avoided injury to the nasal mucosa in the microscopic transseptal approach. That is, the transseptal approach has a great potential with excellent nasal outcomes when manipulating nasal tissue carefully.

Therefore, the endoscopic endonasal transseptal approach can be deemed as a favorable technique with the advantages of both approaches: comparable neurosurgical outcomes from the endoscopic transnasal approach and minimal nasal morbidities from the microscopic transseptal approach. Previous studies reported solely on either neurosurgical or rhinological outcomes. Favier et al. reported similar gross total resection, endocrine outcomes and rates of neurosurgical complication in endoscopic transseptal approach comparing to results of endoscopic transnasal approach from other experienced teams. ${ }^{4}{ }^{5} \mathrm{Hong}$ et al. reported fewer subjective nasal symptoms in the early postoperative period after the endoscopic transseptal approach. ${ }^{6}$ This study demonstrated lower SNOT-22 scores, lower postoperative Lund-Kennedy scores and intact olfactory function in the transseptal group. Meanwhile the neurosurgical outcome and rates of complications were not inferior. That is, the endoscopic transseptal approach enhances nasal recovery without compromising resection, making it a worthy tradeoff for better rhinological outcomes.

We observed that olfactory function was well preserved in both groups. The possible explanation is that olfactory neural fibers was not disrupted in either approaches. ${ }^{23}$ In the transseptal approach, the incisions are made anteriorly and the olfactory nerves can be retained within the elevated mucosal flaps. The binostril method also allows for less tension in the mucosal flap, preventing an unbalanced force from disrupting the olfactory neuroepithelium, as in microscopic TSA. ${ }^{24}$ For the transnasal approach, the main route includes the posterior septum and spared the main olfactory neuroepithelium from permanent dysfunction. ${ }^{25}$

Some may concern that managing unexpected high-flow intraoperative CSF leakage could be problematic for the endoscopic transseptal TSA. On the contrary, a nasoseptal flap can be harvested by dividing the septal mucosal flap superiorly and inferiorly. For patients with a high risk of CSF leakage, the septal incision can be made anteriorly to develop a larger nasoseptal flap.

Even though the endoscopic transseptal approach seems attractive, surgeons should remember that every procedure has its limitations. Dissection of the septal mucosal flap could be difficult in patients with previous nasal surgery including TSA, septoplasty and rhinoplasty. In addition, tumors with lateral extension to the cavernous sinus cannot be removed thoroughly. Finally, although septal infection presented only in one patient in our series, severe septal infection may result in perforation or nasal deformity. We should carefully examined patients with unusual postoperative nasal pain.

There were several limitations in this study. First, the study was retrospective in nature. Although we have controlled as many confounding factors as possible, the results still require validation by a prospective randomised controlled studies. Second, we included only pituitary adenoma and whether the conclusions can be generalised to other sellar pathologies remains unexplored. Finally, we only analysed data until 1 year after surgery. Long-term complications, especially for septal complications, require a longer follow-up.

\section{CONCLUSIONS}

The transseptal approach is an effective procedure for pituitary adenoma with better nasal recovery. It might be considered as an alternative surgical technique for pituitary adenoma under selected circumstances.

\section{REFERENCES}


1. Lee JT, Kingdom TT, Smith TL, Setzen M, Brown S, Batra PS. Practice patterns in endoscopic skull base surgery: survey of the American Rhinologic Society. Int Forum Allergy Rhinol. 2014;4(2):124-31.

2. Lee JY, Bohman LE, Bergsneider M. Contemporary neurosurgical techniques for pituitary tumor resection. J Neurooncol. 2014;117(3):437-44.

3. Asemota AO, Ishii M, Brem H, Gallia GL. Comparison of Complications, Trends, and Costs in Endoscopic vs Microscopic Pituitary Surgery: Analysis From a US Health Claims Database. Neurosurgery. 2017;81(3):458-72.

4. Favier V, Boetto J, Cartier C, Segnarbieux F, Crampette L. Endoscopic transnasal transseptal pituitary surgery. Eur Ann Otorhinolaryngol Head Neck Dis. 2019;136(2):131-4.

5. Favier V, Le Corre M, Segnarbieux F, Rigau V, Raingeard I, Cartier C, et al. Endoscopic subperichondrial transseptal transsphenoidal approach is safe and efficient for non-extended pituitary surgery. Eur Arch Otorhinolaryngol. 2020;277(4):1079-87.

6. Hong SD, Nam DH, Kong DS, Kim HY, Chung SK, Dhong HJ. Endoscopic Modified Transseptal Transsphenoidal Approach for Maximal Preservation of Sinonasal Quality of Life and Olfaction. World Neurosurg. 2016;87:162-9.

7. Hong SD, Nam DH, Seol HJ, Choi NY, Kim HY, Chung SK, et al. Endoscopic binostril versus transnasal transseptal microscopic pituitary surgery: Sinonasal quality of life and olfactory function. Am J Rhinol Allergy. 2015;29(3):221-5.

8. Wen G, Tang C, Zhong C, Li J, Cong Z, Zhou Y, et al. One-and-a-half nostril endoscopic transsphenoidal approach for pituitary adenomas-a technical report. J Otolaryngol Head Neck Surg. 2016;45(1):60.

9. Sullivan CB, Schwalje AT, Jensen M, Li L, Dlouhy BJ, Greenlee JD, et al. Cerebrospinal Fluid Leak After Nasal Swab Testing for Coronavirus Disease 2019. JAMA Otolaryngol Head Neck Surg. 2020.

10. Hanna AS, Grindle CR, Patel AA, Rosen MR, Evans JJ. Inadvertent insertion of nasogastric tube into the brain stem and spinal cord after endoscopic skull base surgery. Am J Otolaryngol. 2012;33(1):178-80.

11. Wu V, Cusimano MD, Lee JM. Extent of surgery in endoscopic transsphenoidal skull base approaches and the effects on sinonasal morbidity. Am J Rhinol Allergy. 2018;32(1):52-6.

12. Hsieh CH, Chen PG, Zhou B, Lin LJ, Lai JT, Shen PH. Investigation of Normative Value of Commercialized Taiwan Smell Identification Test. Allergy Rhinol (Providence). 2021;12:2152656721991525.

13. Little AS, Kelly D, Milligan J, Griffiths C, Prevedello DM, Carrau RL, et al. Predictors of sinonasal quality of life and nasal morbidity after fully endoscopic transsphenoidal surgery. J Neurosurg. 2015;122(6):145865.

14. Shay A, Sturgis M, Ritz EM, Beer-Furlan A, Munoz L, Byrne R, et al. Prior smoking and nasoseptal flap usage adversely impact quality of life and healing after endoscopic pituitary surgery. Neurosurg Focus. 2020;48(6):E17.

15. Soyka MB, Serra C, Regli L, Meier E, Holzmann D. Long-term olfactory outcome after nasoseptal flap reconstructions in midline skull base surgery. Am J Rhinol Allergy. 2017;31(5):334-7.

16. Rioja E, Bernal-Sprekelsen M, Enriquez K, Ensenat J, Valero R, de Notaris M, et al. Long-term outcomes of endoscopic endonasal approach for skull base surgery: a prospective study. Eur Arch Otorhinolaryngol. 2016;273(7):1809-17.

17. Hong SD, Nam DH, Park J, Kim HY, Chung SK, Dhong HJ. Olfactory outcomes after endoscopic pituitary surgery with nasoseptal "rescue" flaps: electrocautery versus cold knife. Am J Rhinol Allergy. 2014;28(6):517-9. 
18. Almutairi RD, Muskens IS, Cote DJ, Dijkman MD, Kavouridis VK, Crocker E, et al. Gross total resection of pituitary adenomas after endoscopic vs. microscopic transsphenoidal surgery: a meta-analysis. Acta Neurochir (Wien). 2018;160(5):1005-21.

19. Castano-Leon AM, Paredes I, Munarriz PM, Jimenez-Roldan L, Hilario A, Calatayud M, et al. Endoscopic Transnasal Trans-Sphenoidal Approach for Pituitary Adenomas: A Comparison to the Microscopic Approach Cohort by Propensity Score Analysis. Neurosurgery. 2020;86(3):348-56.

20. Li A, Liu W, Cao P, Zheng Y, Bu Z, Zhou T. Endoscopic Versus Microscopic Transsphenoidal Surgery in the Treatment of Pituitary Adenoma: A Systematic Review and Meta-Analysis. World Neurosurg. 2017;101:236-46.

21. Cho J, Grayson JW, Christensen J, Winder MJ, Sheehy J, Steel T, et al. Long-Term Sinonasal Function Following Transnasal Pituitary Surgery: A Comparison of Surgical Approach. Am J Rhinol Allergy. 2020;34(3):361-8.

22. Pledger CL, Elzoghby MA, Oldfield EH, Payne SC, Jane JA, Jr. Prospective comparison of sinonasal outcomes after microscopic sublabial or endoscopic endonasal transsphenoidal surgery for nonfunctioning pituitary adenomas. J Neurosurg. 2016;125(2):323-33.

23. Patel ZM, DelGaudio JM. Olfaction following endoscopic skull base surgery. Curr Opin Otolaryngol Head Neck Surg. 2016;24(1):70-4.

24. Majovsky M, Astl J, Kovar D, Masopust V, Benes V, Netuka D. Olfactory function in patients after transsphenoidal surgery for pituitary adenomas-a short review. Neurosurg Rev. 2019;42(2):395-401.

25. Yin LX, Low CM, Puccinelli CL, O'Brien EK, Stokken JK, Van Abel KM, et al. Olfactory outcomes after endoscopic skull base surgery: A systematic review and meta-analysis. Laryngoscope. 2019;129(9):19982007.

\section{FIG. LEGENDS}

Fig. 1. Transseptal approach started from posterior septectomy after elevating septal flaps (A), with olfactory nerves protected (B; arrowhead: olfactory nerves). The rostrum was removed (C), followed by tumor resection until the arachnoid membrane descended (D).

Fig. 2. Postoperative Lund-Kennedy scores (A) and 22-item sinonasal outcome test (SNOT-22) scores (B) were significantly lower in the transseptal group, while olfactory test revealed no significant difference (C). ${ }^{*} P<.05 ;{ }^{* *} P<.01 ;{ }^{* * *} P<.001$

\section{VIDEO LEGENDS}

Video 1 . Intraoperative video demonstrated surgical procedures of the endoscopic transseptal approach.

\section{Hosted file}

Table 0117.docx available at https://authorea.com/users/456846/articles/553815-betternasal-recovery-and-intact-olfactory-function-after-transseptal-approach-for-endoscopicendonasal-transphenoidal-adenomectomy-a-retrospective-analysis 

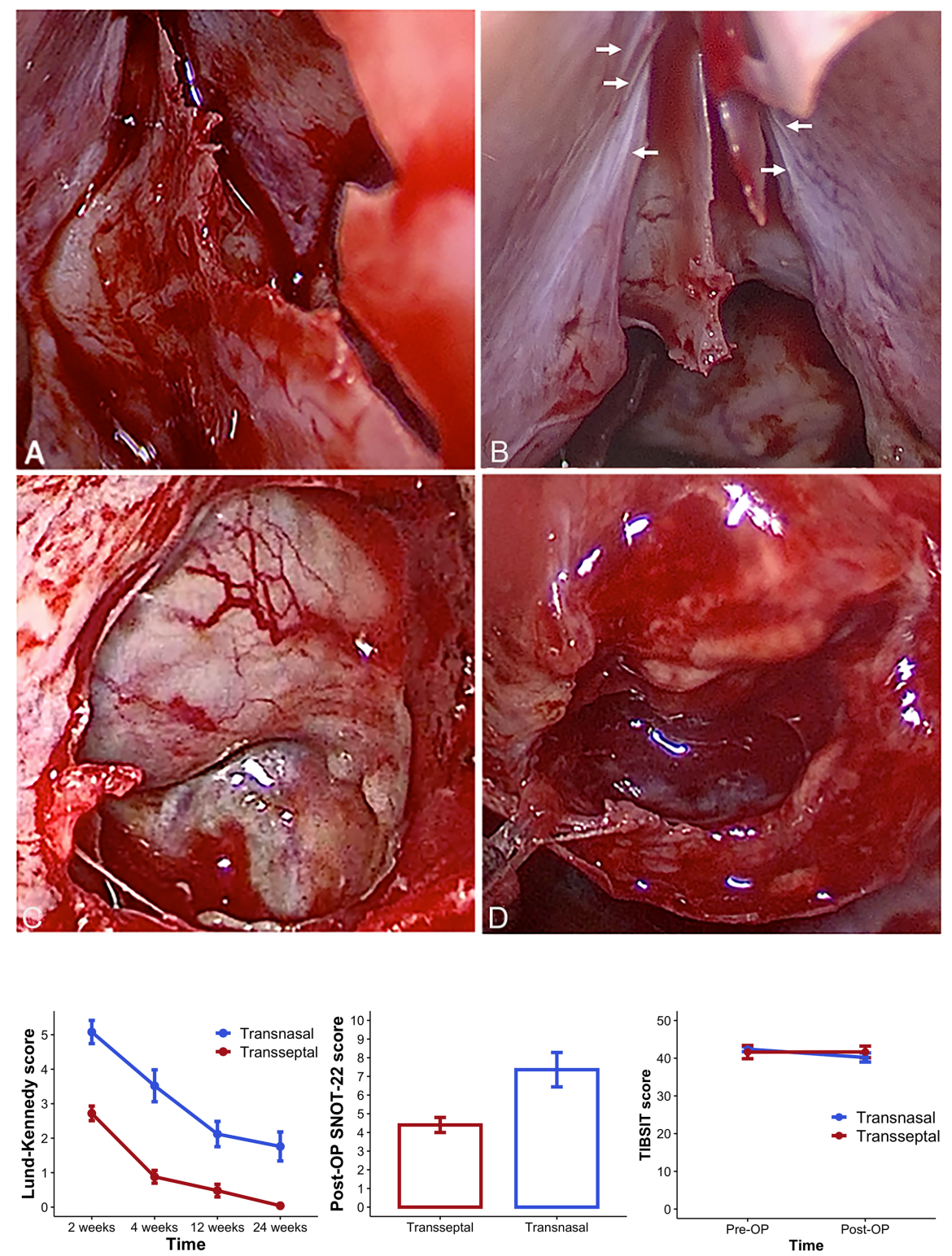